\title{
An Artificial Immune System Approach for B-Spline Surface Approximation Problem
}

\author{
Erkan Ülker ${ }^{1}$ and Veysi İşler ${ }^{2}$ \\ ${ }^{1}$ Selçuk University, Department of Computer Engineering, 42075 Konya, Turkey \\ eulker@selcuk.edu.tr \\ ${ }^{2}$ Middle East Technical University, Department of Computer Engineering, 06531 Ankara, \\ Turkey \\ isler@ceng.metu.edu.tr
}

\begin{abstract}
In surface fitting problems, the selection of knots in order to get an optimized surface for a shape design is well-known. For large data, this problem needs to be dealt with optimization algorithms avoiding possible local optima and at the same time getting to the desired solution in an iterative fashion. Many computational intelligence optimization techniques like evolutionary optimization algorithms, artificial neural networks and fuzzy logic have already been successfully applied to the problem. This paper presents an application of another computational intelligence technique known as "Artificial Immune Systems (AIS)" to the surface fitting problem based on BSplines. Our method can determine appropriate number and locations of knots automatically and simultaneously. Numerical examples are given to show the effectiveness of our method. Additionally, a comparison between the proposed method and genetic algorithm is presented.
\end{abstract}

\section{Introduction}

Since B-spline curve fitting for noisy or scattered data can be considered as a nonlinear optimization problem with high level of computational complexity [3, 4, 6], non-deterministic optimization strategies should be employed. Here, methods taken from computational intelligence offers promising results for the solutions of this problem. By the computational intelligence techniques as is utilized in this paper, we mean the strategies that are inspired from numerically based Artificial Intelligence systems such as evolutionary algorithms and neural networks. One of the most conspicuous and promising approaches to solve this problem is based on neural networks. Previous studies are mostly focused on the traditional surface approximation [1] and the first application of neural networks to this field is taken place in [15]. Later on, the studies that include Kohonen networks [8, 9, and 12], SelfOrganized Maps [13, 14] and Functional networks [5, 7, and 10] provided extension of studies of surface design. Evolutionary algorithms are based on natural selection for optimization with multi-aims. Most of the evolutionary optimization techniques such as Genetic Algorithm (GA) [3, 6, and 17], Simulated Annealing [16] and Simulated Evolution [17, 18, and 19] are applied to this problem successfully. 
This paper presents the application of one of the computational intelligence techniques called "Artificial Immune System (AIS)" to the surface fitting problem by using B-Splines. Individuals are formed by treating knot placement candidates as antibody and the continuous problem is solved as a discrete problem as in [3] and [6]. By using Akaike Information Criteria (AIC), affinity criterion is described and the search is implemented from the good candidate models towards the best model in each generation. The proposed method can describe the placement and numbers of the knot automatically and concurrently. In this paper, the numerical examples are given to show the effectiveness of the proposed method. Moreover, a comparison between the proposed method and the genetic algorithm is presented.

\section{B-Spline Surface Approximation}

Geometry fitting can be formulized as the minimization of the fitting error under some accuracy constraints in the jargon of mathematics. A typical error scale for parametrical surface fitting is as follows;

$$
Q_{2}=\sum_{i=1}^{N_{N}} \sum_{j=1}^{N_{y}} w_{i, j}\left\{S\left(x_{i}, y_{j}\right)-F_{i, j}\right\}^{2}
$$

Surface fitting from sample points is also known as surface reconstruction. This paper applies a local fitting and blending approach to this problem. The readers can refer to [1] and [2] for details. A B-Spline curve, $C(u)$, is a vector valued function which can be expressed as:

$$
C(u)=\sum_{i=0}^{m} N_{i, k}(u) P_{i}, \quad u \in\left[u_{k-1}, u_{m+1}\right]
$$

where $P_{i}$ represents control points (vector) and $N_{i, k}$ is the normal $k$-degree B-spline basis functions and $N_{i, k}$ can be defined as a recursive function as follows.

$$
N_{i, 1}(u)=\left\{\begin{array}{ll}
1 & \text { if } u \in\left[u_{i}, u_{i+1}\right), \\
0 & \text { otherwise }
\end{array} \quad \text { and } \quad N_{i, k}(u)=\frac{u-u_{i}}{u_{i+k-1}-u_{i}} N_{i, k-1}(u)+\frac{u_{i+k}-u}{u_{i+k}-u_{i+1}} N_{i+1, k-1}(u)\right.
$$

where $u_{i}$ represents knots that are shaped by a knot vector and $U=\left\{u_{0}, u_{1}, \ldots, u_{m}\right\}$. Any B-Spline surface is defined as follows:

$$
S(u, v)=\sum_{i=0}^{m} \sum_{j=0}^{n} N_{i, k}(u) * N_{j, l}(v) * P_{i, j}
$$

As can be seen from upper equations, B-spline surface is given as unique with the degree, knot values and control points. Then, surface is formed by these parameters. Input is a set of unorganized points in surface reconstruction problems so the degree of the surface, knots and control points are all unknown. In equation (3), the knots not only exist in the dividend but also exist in the denominator of the fractions. Thus a spline surface given as in equation (4) is a function of nonlinear knots. Assume that 
the data to be fitted are given as if they are on the mesh points of the $D=[a, b] x[c, d]$ rectangular field on the $x-y$ plane. Then the expression like below can be written [3]:

$$
F_{i, j}=f\left(x_{i}, y_{j}\right)+\varepsilon_{i, j}, \quad\left(i=1,2, \cdots, N_{x} ; j=1,2, \cdots, N_{y}\right) .
$$

In this equation, $f(x, y)$ is the function exists at the base of data, $N_{x}$ and $N_{y}$ represent the number of the data points in $x$ and $y$ directions respectively and $\varepsilon_{i, j}$ is a measurement error. Equation (4) is adjusted or fitted to given data by least square methods with equation (5). For parameterization of B-spline curve in Equation (2) and surface in Equation (4) must be produced by preferring one of the parameterization methods among uniform, chordal or centripetal methods. Then, the squares of remainders or residuals are calculated by Equation (1). The lower index of the $Q_{2}$ means the dimension of the data. The objective that will be minimized in B-spline surface fitting problem is the function in Equation (1) and the variables of the object function are B-spline coefficients and interior knots. B-spline coefficients are linear parameters. However, interior knots are nonlinear parameters since $S(u, v)$ function is a nonlinear knots function. This minimization problem is known as multi-modal optimization problem [4].

\section{B-Spline Surface Approximation by Artificial Immune System}

AIS were emerged as a new system that combines varieties of biological based computational methods such as Artificial Neural Network and Artificial Life in 1990s. AIS were used in very diverse areas such as classification, learning, optimization, robotic and computer security [11]. We need the following components to construct the AIS: (i) The representation of system parts, (ii) A mechanism to compute the interaction of system parts with each other and with the environment, (iii) Adoption procedures. Different methods were employed for each of these components in the algorithms that have been developed until now. We decided that Clonal Selection algorithm was the best for the purpose of our study.

Distance criterion is used for determination of mutual interaction degree of Antigen-Antibody as a scale. If antibody and antigen are represented as $A b=<\mathrm{Ab}_{1}, \mathrm{Ab}_{2}, \ldots, \mathrm{Ab}_{\mathrm{L}}>$ and $A g=<\mathrm{Ag}_{1}, \mathrm{Ag}_{2}, \ldots, \mathrm{Ag}_{\mathrm{L}}>$, respectively, Euclid distance between $A b$ and $A g$ is calculated as follows:

$$
D=\sqrt{\sum_{i=1}^{L}\left(A b_{i}-A g_{i}\right)^{2}}
$$

B-spline surface fitting problem is to approximate the B-spline surface that approximate a target surface in a certain tolerance interval. Assume that object surface is defined as $N_{x} x N_{y}$ grid type with ordered and dense points in 3D space and the knots of the B-spline surface that will be fitted are $n_{x} x n_{y}$ grid that is subset of $N_{x} x N_{y}$ grid. Degrees of curves, $m_{x}$ and $m_{y}$, will be entered from the user. Given number of points $N_{x} x N_{y}$ is assigned to $L$ that is dimensions of the Antigen and Antibody. Each bit of Antibody and Antigen is also called their molecule and is equivalent to a data point. If the value of a molecule is $l$ in this formulation then a knot is placed to a suitable data point otherwise no knot is placed. If the given points are lied down 
between $[a, b]$ and $[c, d]$ intervals, $n_{x} x n_{y}$ items of knots are defined in this interval and called as interior knots. Initial population includes $k$ Antibody with $L$ numbers of molecules. Molecules are set as 0 and 1, randomly.

For recognizing (response against Antigen) process, affinity of Antibody against Antigen were calculated as in Equation (7) that uses the distance between AntibodyAntigen and AIC that is preferred as fitness measure in [3] and [6] references.

$$
\text { Affinity }=1-\left(\text { AIC } / \text { Fitness }_{\text {avrg }}\right)
$$

In Equation (7), Fitness ${ }_{\text {avrg }}$ represents the arithmetical average of AIC values of all Antibodies in the population and calculated as follow. If the AIC value of any of the individual is greater than Fitness $s_{\text {avrg }}$ then Affinity is accepted as zero (Affinity=0) in Equation (7).

$$
\text { Fitness }_{\text {avrg }}=\sum_{i=1}^{K} A I C_{i} / K
$$

Where, $K$ is the size of the population and $A I C_{i}$ is the fitness measure of the $i^{\text {th }}$ antibody in the population. AIC is given as below.

$$
A I C_{2}=N_{x} N_{y} \log _{e} Q_{2}+2\left\{\left(n_{x}+m_{x}\right)\left(n_{y}+m_{y}\right)+n_{x}+n_{y}\right\}
$$

The antibody which is ideal solution and the exact complementary of the Antigen is the one whose affinity value is nearest to 1 among the population (in fact in memory). Euclid distance between ideal Antibody and Antigen is equal to zero. In this case the problem becomes not surface approximation but surface interpolation.

In order to integrate clonal selection algorithm to this problem some modification must be carried out on the original algorithm. The followings are step by step explanation of the modifications made on the algorithm and how these modifications applied to above mentioned algorithm.

1. Enter the data points to be fitted (In a form of $N_{x}$ and $N_{y}$ grid).

2. Enter the control parameters.

3. Build initial Antibody population with random molecules.

4. If the population is built as the first time, create memory array (save all antibodies).

5. Otherwise, update antibody population and memory cells and develop the memory.

6. For each of antibody calculate B-spline and fit it to the given curve. Later on calculate the sum of squares of residuals $\left(Q_{2}\right)$.

7. For each of antibody in the population calculate its AIC value and calculate the average AIC value of population.

8. For each of antibody calculate the affinity.

9. Choose the best antibody according to the affinity and in request antigen and interactions of every antibody. The number of the clones will be $K$-varieties.

10. Produce matured antibody population by making the molecules change rational with affinity values of clones.

11. Implement mutation according to the mutation rate.

12. Produce new antibody according to the variety ratio.

13. If iteration limit is not reached or antigen is not recognized fully go to step 5 . 


\section{Experimental Results}

In order to evaluate the proposed AIS based Automatic Knot Placement algorithm five bivariate test functions were used (see Table 1). These functions were constructed to have a unit standard deviation and a non-negative range. Since the antibodies with highest affinity values are kept in the memory in AIS architecture, the antibody of the memory with the highest affinity for each generation was presented in the results. To see the performance evaluation and approximation speed, genetic algorithm suggested by Sarfaz at al. $[6,17]$ and proposed algorithm in this study were compared. Knot ratio and operation of making important points immobilize at the knot chromosome are discarded in their algorithm. The developed program has the flexibility of entering the B-spline surface orders from the user. To test the quality of the proposed model, Root Mean Square $(R M S)$ error were calculated for $M$ and $N$ values from 5 to 10 for 400 (20x20) training data points from five surfaces defined as above. Initial population is fed till 500 generations. Increase in the number of generation makes increase in fitting (error decreases). The slot of the approximated curves indicates the probability of having still approximation in next generation. Table 2 shows the statistics of $G A$ and $A I S$ optimization execution. RMS errors between point clouds and modeled surface based on the best chromosome in $G A$ and antibodies in population of memory in AIS were given in Table 3 for four test functions (Surface I-Surface IV).

The analyses are implemented for all surfaces in Table 1. M $M N$ mesh is determined as randomly for Surface II - Surface IV. At Surface II shown in Table 3, the choices for $M$ and $N$ are fitting to $M x N=8 x 8$. Similarly, the choices of $M$ and $N$ for Surface $I I I$ and $I V$ are fitting to $M x N=9 x 9$ and $M x N=10 x 10$, respectively.

Table 1. Five test functions for the bivariate setting

\begin{tabular}{l}
\hline$f\left(x_{1}, x_{2}\right)=10.391\left\{\left(x_{1}-0.4\right)\left(x_{2}-0.6\right)+0.36\right\}$ \\
$f\left(x_{1}, x_{2}\right)=24.234\left\{r^{2}\left(0.75-r^{2}\right)\right\}, \quad r^{2}=\left(x_{1}-0.5\right)^{2}+\left(x_{2}-0.5\right)^{2}$ \\
$f\left(x_{1}, x_{2}\right)=42.659\left\{0.1+\overline{x_{1}}\left(0.05+\bar{x}_{1}^{4}-10 \bar{x}_{1}^{2} \bar{x}^{2}+5 \bar{x}_{4}^{4}\right)\right\} \overline{x_{1}}=x_{1}-0.5, \overline{x_{2}}=x_{2}-0.5$ \\
$\left.f\left(x_{1}, x_{2}\right)=1.3356 \mid 1.5\left(1-x_{1}\right)+e^{2 x_{1}-1} \operatorname{Sin}\left\{3 \pi\left(x_{1}-0.6\right)^{2}\right\}+e^{3\left(x_{2}-0.5\right)} \operatorname{Sin}\left\{4 \pi\left(x_{2}-0.9\right)^{2}\right\}\right]$ \\
$f\left(x_{1}, x_{2}\right)=1.9\left[1.35+e^{x_{1}} \operatorname{Sin}\left\{13\left(x_{1}-0.6\right)^{2}\right\}+e^{x_{2}} \operatorname{Sin}\left(7 x_{2}\right)\right]$ \\
\hline
\end{tabular}

Table 2. Parameter Set

\begin{tabular}{lll}
\hline Parameter & AIS & GA \\
\hline Mesh Size & $20 \times 20$ & $20 \times 20$ \\
Population size & 20 & 20 \\
String length & 200 (Antibody cell length) & 200 (chromosome gen-length) \\
Mutation Rate & None & 0.001 \\
Crosover & None & 0.7 \\
Variety & $6(30 \%)$ & $6(30 \%)$ \\
Memory Size & 40 & None \\
Generation & 500 & 500 \\
B-spline's order & Random and user defined & Random and user defined \\
\hline
\end{tabular}


Table 3. RMS $\left(x 10^{-2}\right)$ values of AIS and GA methods for 400 data points from Surface I to Surface IV for different MxN

\begin{tabular}{ccccccccc}
\hline Gen. & \multicolumn{2}{c}{$\begin{array}{c}\text { Surface I (7x7) } \\
\text { G.A. }\end{array}$} & \multicolumn{2}{c}{ A.I.S. } & \multicolumn{2}{c}{ G.A. } & A.I.S. & \multicolumn{2}{c}{ G.A. A.I.S. } & \multicolumn{2}{c}{ G.A. A. } & A.I.S. \\
\hline 1 & 8.26 & 9.34 & 3.64 & 3.69 & 10.10 & 10.25 & 8.21 & 8.57 \\
10 & 7.99 & 7.14 & 3.70 & 3.59 & 11.25 & 9.57 & 8.76 & 8.02 \\
25 & 8.22 & 5.88 & 4.25 & 3.42 & 9.67 & 8.71 & 8.36 & 7.61 \\
50 & 8.72 & 5.61 & 3.91 & 2.86 & 10.40 & 7.93 & 8.53 & 7.44 \\
100 & 8.34 & 5.53 & 3.72 & 2.71 & 9.97 & 7.48 & 8.78 & 7.22 \\
200 & 8.72 & 5.58 & 4.01 & 2.01 & 10.50 & 7.06 & 9.30 & 7.10 \\
300 & 8.99 & 5.29 & 4.60 & 1.58 & 10.61 & 7.03 & 7.99 & 7.07 \\
400 & 7.63 & 5.23 & 3.52 & 1.52 & 10.38 & 7.03 & 8.45 & 7.00 \\
500 & 8.86 & 5.23 & 3.99 & 1.50 & 10.60 & 7.00 & 8.57 & 6.95 \\
\hline
\end{tabular}

Table 4. RMS $\left(\times 10^{-2}\right)$ values of AIS and GA methods for 400 data points from Surface V for different MxN. (a) AIS, and (b) GA.

\begin{tabular}{lllllllll}
\hline & M x N & 5 & & 6 & 7 & 8 & 9 & 10 \\
\hline AIS & 5 & 8.6015 & 8.0171 & 7.9324 & 7.0035 & 7.3263 & 7.0629 \\
& 6 & 8.4809 & 8.4179 & 8.3722 & 7.5269 & 7.3554 & 7.1965 \\
& 7 & 7.6333 & 7.2749 & 7.4622 & 7.2034 & 6.9558 & 6.1804 \\
& 8 & 7.8404 & 6.8614 & 6.4382 & 6.4288 & 6.7375 & 6.0138 \\
& 9 & 7.9077 & 7.8398 & 6.9039 & 6.9028 & 6.8971 & 5.7911 \\
& 10 & 8.0664 & 6.7625 & 7.1614 & 6.3575 & 6.9640 & 6.3637 \\
GA & 5 & 10.501 & 9.6512 & 9.1281 & 9.7179 & 9.8944 & 9.6573 \\
& 6 & 10.238 & 9.8221 & 9.3189 & 9.5761 & 7.7725 & 8.5993 \\
& 7 & 9.9913 & 9.4922 & 8.9494 & 8.2377 & 8.1184 & 8.1649 \\
& 8 & 10.013 & 8.6365 & 8.6247 & 8.2134 & 7.6657 & 7.8947 \\
& 9 & 10.020 & 9.1249 & 8.7523 & 8.1843 & 7.3076 & 7.4484 \\
& 10 & 9.3970 & 9.1297 & 8.4642 & 8.2721 & 7.6331 & 7.3366 \\
\hline
\end{tabular}

Table 5. Fitness and RMS statistics of GA and AIS for Surface V

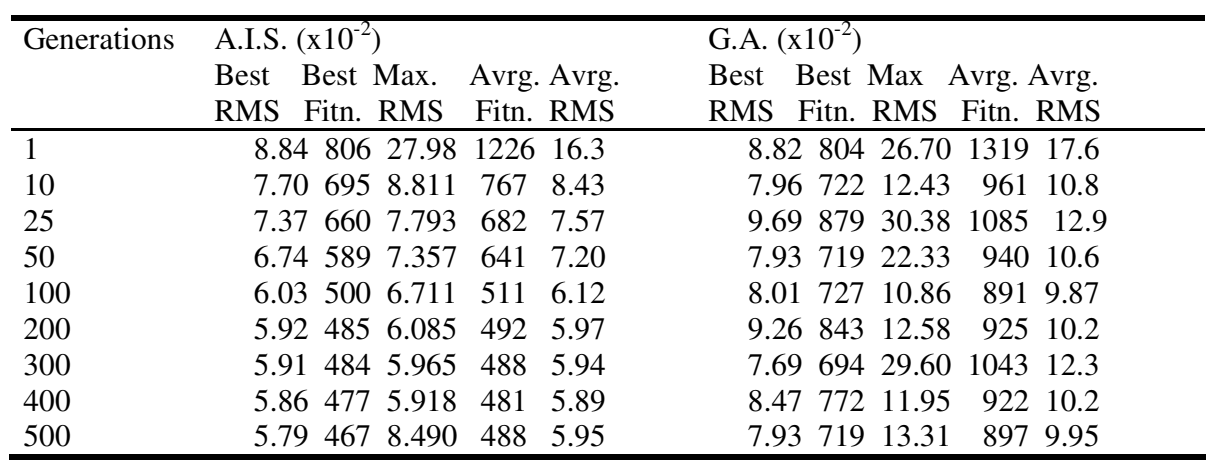

Table 4 points out Surface $V$. RMS error was calculated for some $M$ and $N$ values from 5 to 10 for $400(20 \times 20)$ training data points. As the reader can evaluate, the error approaches depending on the $M$ and $N$ values are reasonable and the best choice fits to the $M x N=9 x 10$ as shown italic in Table 4. According to the knots $M x N$ that offer best 
fitting, the proposed algorithm was also compared with $G A$ based algorithm by Safraz et al. regarding to approximation speed. The outputs of the programs were taken for some generations in the training period. Best and average fitness values of individuals and antibodies according to the related generations of the program outputs were given in Table 5. The graphics of approximations of the proposed AIS approach and GA approach for all generations are given in Fig. 1. The bold line and dotted line represent maximum fitness and average fitness values respectively.
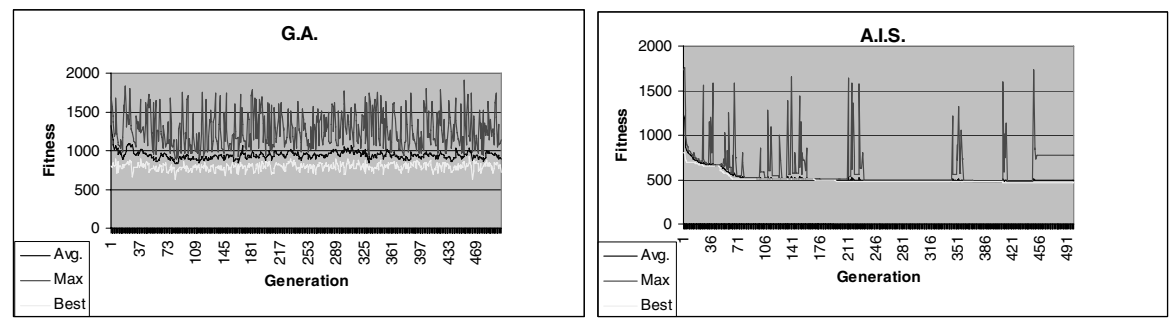

Fig. 1. Parameter optimization based on GA and AIS regarding to the generations

\section{Conclusion and Future Work}

This paper presents an application of another computational intelligence technique known as "Artificial Immune Systems (AIS)" to the surface fitting problem using Bsplines. In this study, original problem like in [3] and [6] was converted to a discrete combinational optimization problem and solved as the strategy of this study. In this paper, it has been clearly shown that proposed AIS algorithm is very useful for the recognition of the good knot automatically. The suggested method can describe the numbers and placements of the knots concurrently. None of the subjective parameters such as error tolerance or a regularity (order) factor and initial places of the knots that are chosen well are not required.

There are two basic requirements on each of B-spline surface in iterations for guaranteeing the appropriate approximation in B-spline surface fitting. (1) Its shape must be similar to the target or object surface; (2) its control points must be scattered or its knot points must be determined appropriately. The technique presented in this paper is proved to reduce the necessity of the second requirement.

In this study, Clonal Selection Algorithm of AIS is applied to the surface reconstruction problem and various new ways of surface modeling is developed. The big potential of this approach has been shown. For a given set of 3D data points, AIS assists to choice the most appropriate B-spline surface degree and knot points. The authors of this manuscript will use other AIS technique to improve the proposed method in their future studies. The positive or negative effects of other techniques will tried to be obtained and comparisons will be done in the future studies. Additionally, NURBS surfaces will be used to improve the suggested algorithm. This extension is especially important regarding to have the complex optimization of weight of NURBS. 


\section{Acknowledgements}

This study has been supported by the Scientific Research Projects of Selcuk University (in Turkey).

\section{References}

1. Weiss, V., Andor, L., Renner, G., Varady, T., Advanced surface fitting techniques, Computer Aided Geometric Design Vol. 19, p. 19-42, (2002).

2. Piegl, L., Tiller, W., The NURBS Book, Springer Verlag, Berlin, Heidelberg, (1997).

3. Yoshimoto, F., Moriyama, M., Harada, T., Automatic knot placement by a genetic algorithm for data fitting with a spline, Proc. of the International Conference on Shape Modeling and Applications, IEEE Computer Society Press, pp. 162-169, (1999).

4. Goldenthal, R., Bercovier, M. Design of Curves and Surfaces by Multi-Objective Optimization, April 2005, Leibniz Report 2005-12.

5. Iglesias, A., Echevarr'ýa, G., Galvez, A., Functional networks for B-spline surface reconstruction, Future Generation Computer Systems, Vol. 20, pp. 1337-1353, (2004).

6. Sarfraz, M., Raza, S.A., Capturing Outline of Fonts using Genetic Algorithm and Splines, Fifth International Conference on Information Visualisation (IV'01), pp. 738-743, (2001).

7. Iglesias, A., Gálvez, A., A New Artificial Intelligence Paradigm for Computer-Aided Geometric Design, Lecture Notes in Artificial Intelligence Vol. 1930, pp. 200-213, (2001).

8. Hoffmann, M., Kovács E., Developable surface modeling by neural network, Mathematical and Computer Modelling, Vol. 38, pp. 849-853, (2003)

9. Hoffmann, M., Numerical control of Kohonen neural network for scattered data approximation, Numerical Algorithms, Vol. 39, pp. 175-186, (2005).

10. Echevarría, G., Iglesias, A., Gálvez, A., Extending Neural Networks for B-spline Surface Reconstruction, Lecture Notes in Computer Science, Vol. 2330, pp. 305-314, (2002).

11. Engin, O., Döyen, A., Artificial Immune Systems and Applications in Industrial Problems, Gazi University Journal of Science 17(1): pp. 71-84, (2004).

12. Boudjemaï, F., Enberg, P.B., Postaire, J.G., Surface Modeling by using Self Organizing Maps of Kohonen, IEEE Int. Conf. on Systems, Man and Cybernetics, vol. 3, pp. 2418-2423, (2003).

13. Barhak, J., Fischer, A., Adaptive Reconstruction of Freeform Objects with 3D SOM Neural Network Grids, Journal of Computers \& Graphics, vol. 26, no. 5, pp. 745-751, (2002).

14. Kumar, S.G., Kalra, P. K. and Dhande, S. G., Curve and surface reconstruction from points: an approach based on SOM, Applied Soft Computing Journal, Vol. 5(5), pp. 55-66, (2004).

15. Hoffmann, M., Várady, L., and Molnar, T., Approximation of Scattered Data by Dynamic Neural Networks, Journal of Silesian Inst. of Technology, pp, 15-25, (1996).

16. Sarfraz, M., Riyazuddin, M., Curve Fitting with NURBS using Simulated Annealing, Applied Soft Computing Technologies: The Challenge of Complexity, Series: Advances in Soft Computing, Springer Verlag, (2006).

17. Sarfraz, M., Raza, S.A., and Baig, M.H., Computing Optimized Curves with NURBS Using Evolutionary Intelligence, Lect. Notes in Computer Science, Volume 3480, pp. 806-815, (2005).

18. Sarfraz, M., Sait, Sadiq M., Balah, M., and Baig, M. H., Computing Optimized NURBS Curves using Simulated Evolution on Control Parameters, Applications of Soft Computing: Recent Trends, Series: Advances in Soft Computing, Springer Verlag, pp. 35-44, (2006).

19. Sarfraz, M., Computer-Aided Reverse Engineering using Simulated Evolution on NURBS, Int. J. of Virtual \& Physical Prototyping, Taylor \& Francis, Vol. 1(4), pp. 243 - 257, (2006). 